\title{
Effectiveness of off-the-shelf footwear in reducing foot pain in Australian Department of Veterans' Affairs recipients not eligible for medical grade footwear: study protocol for a randomized controlled trial
}

\author{
Hylton B Menz ${ }^{1 *}$, Nicoletta Frescos ${ }^{2}$ and Shannon E Munteanu ${ }^{1,2}$
}

\begin{abstract}
Background: Foot pain is highly prevalent in older people, and in many cases is associated with wearing inadequate footwear. In Australia, the Department of Veterans' Affairs (DVA) covers the costs of medical grade footwear for veterans who have severe foot deformity. However, there is a high demand for footwear by veterans with foot pain who do not meet this eligibility criterion. Therefore, this article describes the design of a randomized controlled trial to evaluate the effectiveness of low cost, off-the-shelf footwear in reducing foot pain in DVA recipients who are currently not eligible for medical grade footwear.

Methods: One hundred and twenty DVA clients with disabling foot pain residing in Melbourne, Australia, who are not eligible for medical grade footwear will be recruited from the DVA database, and will be randomly allocated to an intervention group or a 'usual care' control group. The intervention group will continue to receive their usual DVA-subsidized podiatry care in addition to being provided with low-cost, supportive footwear (Dr Comfort ${ }^{\circledR}$, Vasyli Medical, Labrador, Queensland, Australia). The control group will also continue to receive DVA-subsidized podiatry care, but will not be provided with the footwear until the completion of the study. The primary outcome measure will be pain subscale on the Foot Health Status Questionnaire (FHSQ), measured at baseline and 4, 8, 12 and 16 weeks. Secondary outcome measures measured at baseline and 16 weeks will include the function subscale of the FHSQ, the Manchester Foot Pain and Disability Index, the number of DVA podiatry treatments required during the study period, general health-related quality of life (using the Short Form $12^{\circledR}$ Version 2.0), the number of falls experienced during the follow-up period, the Timed Up and Go test, the presence of hyperkeratotic lesions (corns and calluses), the number of participants using co-interventions to relieve foot pain, and participants' perception of overall treatment effect. Data will be analyzed using the intention-to-treat principle.
\end{abstract}

Discussion: This study is the first randomized controlled trial to evaluate the effectiveness of off-the-shelf footwear in reducing foot pain in DVA recipients. The intervention has been pragmatically designed to ensure that the study findings can be implemented into policy and clinical practice if found to be effective.

Trial registration: Australian New Zealand Clinical Trials Registry: ACTRN12612000322831

\footnotetext{
* Correspondence: h.menz@latrobe.edu.au

'Lower Extremity and Gait Studies Program, Faculty of Health Sciences,

La Trobe University, Bundoora 3086, Victoria, Australia

Full list of author information is available at the end of the article
} 


\section{Background}

Foot pain affects approximately one in three people over the age of 65 years [1-3] and has a significant impact on quality of life in this age-group. Studies of older people have demonstrated that foot pain is associated with decreased ability to undertake activities of daily living [4-9], problems with balance and gait $[7,10,11]$ and an increased risk of falls [12-16]. Furthermore, several clinic-based studies assessing health-related quality of life across a range of age-groups have shown that people with foot disorders (such as generalized foot pain [2,17], nail infections [18-20], hallux valgus [21,22] hallux rigidus [23] and plantar heel pain [24]) demonstrate significantly lower scores than those without these conditions. A range of risk factors for foot problems has been identified, including increased age [25-27], female sex $[7,8,26,28]$, obesity $[8,10,28,29]$, and chronic conditions such as osteoarthritis and diabetes $[8,10,29]$.

In addition to these risk factors, there is also strong evidence that many older people wear inappropriate or poor quality footwear, and that ill-fitting footwear may contribute to foot problems. A household survey of people aged over 80 years conducted in the United Kingdom found that most wore slippers all day, irrespective of whether they were housebound [30]. Similarly, a survey of the indoor shoewearing habits of 128 older people in Australia indicated that more than half spent less than 30 Australian dollars on their indoor footwear (most commonly slippers), replaced them infrequently, and often wore their indoor shoes for outdoor activities [31]. More recently, a survey of sub-acute aged care hospital patients reported that only $14 \%$ wore 'safe' footwear, with the most commonly observed detrimental features being a lack of fastening (86\%), slippery soles $(86 \%)$ and an excessively flexible heel counter (77\%) [32].

By far the most commonly encountered problem with footwear in older people is the wearing of shoes that are too small. Burns et al. [33] compared the length and width of the feet and shoes of 65 people aged between 64 and 93 years attending a rehabilitation ward in the United Kingdom, and reported that $72 \%$ wore shoes of an incorrect size. Similarly, a study of 440 Veterans' Affairs patients in the United States reported that only 26\% were found to be wearing appropriately sized shoes [34], and a recent study of 213 people aged 60 to 80 years in Thailand reported that $50 \%$ of women and $34 \%$ of men wore shoes that were too narrow [35]. Several factors may be responsible for this, including fashion influences (particularly in older women [36,37]), not measuring foot dimensions when purchasing shoes [31], or the absence of commercially-available, low-cost footwear that adequately caters for the altered morphology of the elderly foot $[38,39]$. Irrespective of the underlying cause of poorly-fitting footwear, there is evidence that wearing shoes that are too small is associated with foot problems.
In older people, wearing shoes substantially narrower than the foot is associated with corns on the toes, hallux valgus deformity and foot pain, whereas wearing shoes shorter than the foot is associated with lesser toe deformity [40]. Furthermore, a survey of 227 older women revealed that $61 \%$ reported foot pain when wearing shoes (most commonly in the forefoot and toes), and that those with foot pain exhibited a broader forefoot than those without pain [41].

In Australia, the Department of Veterans' Affairs (DVA), as part of the Rehabilitation Appliances Program, covers the costs of medical grade footwear for veterans who have severe foot deformity (defined as feet that cannot be accommodated in regular 'off-the-shelf' footwear). Footwear provision is one of the most common podiatry interventions. An analysis of 1996 to 1997 DVA data indicated that out of the total podiatry DVA population who had received a podiatry intervention $(\mathrm{n}=$ 4,418), 3,227 (73\%) received new medical grade footwear [42]. However, there is also a high demand for footwear by veterans who have foot pain but do not have severely deformed feet. In this context, it is likely that there are a substantial number of veterans who could benefit from the provision of more appropriate footwear. Such an intervention may also reduce the need for frequent, ongoing maintenance foot care provided by podiatrists under the DVA scheme.

Therefore, the aim of this study is to evaluate the effectiveness of relatively low-cost but good quality, custom-fitted footwear to DVA clients with foot pain who do not currently meet the structural deformity criteria for medical grade footwear.

\section{Methods}

The trial has been registered on the Australian New Zealand Clinical Trials Registry (ACTRN12612000322831).

\section{Ethical approval}

The Australian DVA Human Research Ethics Committee provided ethical approval (approval number E012/005 [5.1]) and the La Trobe University Human Ethics Committee formally accepted this approval (E012/004). All participants will provide written informed consent prior to enrolment. Ethical standards will adhere to the $\mathrm{Na}$ tional Health and Medical Research Council (NHMRC) National Statement [43] and the World Medical Association's Declaration of Helsinki [44]. Publications associated with the trial will be formatted according to the Consolidated Standards of Reporting Trials (CONSORT) 2010 statement $[45,46]$.

\section{Design}

This study is a two-group randomized controlled trial with a 16 week prospective follow-up (Figure 1). Participants 


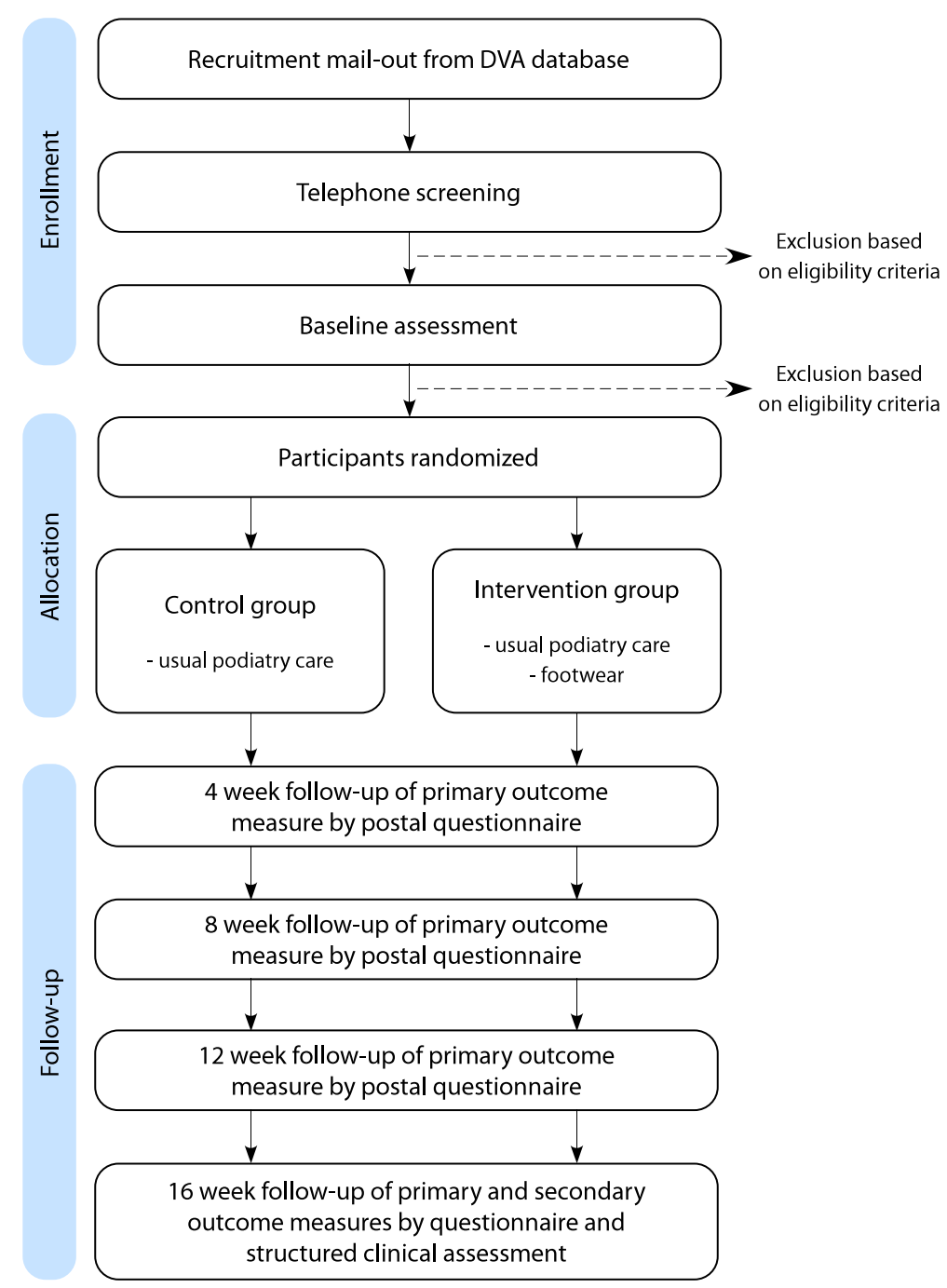

Figure 1 Trial profile.

will be randomly allocated to either a 'usual care' control group or the intervention group [47]. Permuted block randomization with random block sizes (stratified by sex [48]) will be undertaken using an interactive voice response telephone service provided by the NHMRC Clinical Trials Centre at the University of Sydney, New South Wales, Australia to ensure allocation concealment. Due to the nature of the intervention, it will not be possible to blind the participants or investigators. However, the primary outcome measure is administered as a selfcompletion questionnaire and will be entered into the database by an investigator blinded to group allocation.

\section{Participant recruitment, screening and eligibility criteria}

The geocoding feature of the DVA database will be used to identify veterans currently receiving podiatry treatments who are residing in Melbourne, Victoria. From this group of veterans, the DVA Departmental Management
Information System will be used to identify those who have not been issued with medical grade footwear within the last five years, by excluding veterans who have documented a footwear-related item number during this period (item numbers F604, F605, F606, F611, F612, F625, F660, F661 F670, F671, F615 or F616). The remaining veterans will form the primary recruitment source and will be mailed an information package about the study, along with an invitation to contact the research team by either return mail or by telephone. Database recruitment will be complemented by running advertisements in DVA newsletters and placing posters at Returned and Services Leagues Clubs in the surrounding suburbs.

During the initial telephone contact with participants, a member of the research team will determine each veteran's eligibility by structured interview. To be included in the study, veterans will need to meet the following inclusion criteria: 
(i) be aged 65 years or over;

(ii) be a current DVA Gold Card client not eligible for medical grade footwear;

(iii) have received podiatry treatment on at least three occasions in the past five years;

(iv) have disabling foot pain, using the case definition of the Manchester Foot Pain and Disability Index (MFPDI) [49] proposed by Roddy et al. [50]. The MFPDI consists of 19 statements prefaced by the phrase 'Because of pain in my feet', formalized under three constructs: functional limitation (10 items), pain intensity (five items), and personal appearance (two items), with three possible answers: 'none of the time' (score $=0$ ), 'some days' ( The Roddy et al. definition requires at least one of the ten functional limitation items to be documented on most/every day(s) in the last month;

(v) have persistent foot pain, defined as foot pain present for at least 12 weeks, and;

(vi) be capable of understanding the English language in verbal and written form.

Veterans will not be eligible for inclusion in the study if they:

(i) are currently residing in a residential aged care facility;

(ii) have diabetes and a history of foot ulceration (or current foot ulceration) or diabetic peripheral neuropathy (diagnosed with the 5.07 SemmesWeinstein monofilament, using the International Working Group on the Diabetic Foot protocol [51]);

(iii) have a neurodegenerative disorder (for example, Parkinson's disease);

(iv) have had a lower limb or partial foot amputation (although single toe amputations will be permitted);

(v) have been prescribed contoured foot orthoses within the past three months (although simple flat insoles will be permitted, as will contoured foot orthoses prescribed more than three months ago);

(vi) are currently wearing the intervention footwear, or;

(vii) have cognitive impairment (defined as a score of < 7 on the Short Portable Mental Status

Questionnaire [52]).

\section{Intervention group}

The intervention group will continue to receive regular podiatry treatment as clinically required. This will typically include toenail maintenance and scalpel debridement of hyperkeratotic lesions (corns and calluses). In addition, they will be provided with good quality, offthe-shelf footwear (Dr Comfort ${ }^{\circledR}$ Vasyli Medical, Labrador, Queensland, Australia). Men will receive the 'Brian' style and women will receive the 'Annie' style (see Figures 2 and 3). Both styles are available in three width fittings (medium, wide, extra-wide) and feature a stretchable Lycra ${ }^{\circledR}$ upper with Velcro ${ }^{\circledR}$ closure and a removable, flat cushioned insole. These shoes have been selected as they meet all commonly-used criteria for appropriate footwear (such as a low heel, appropriate fixation and adequate depth to accommodate toe deformities) [53-57]. Due to sex differences in foot dimensions (and therefore the dimensions of the lasts the shoes are constructed from) the 'Brian' style has a relatively broader fit than the 'Annie' style. However, both the heel height and toe spring are the same for shoes of equivalent length across the two styles.

The research staff will measure participants' feet using a Brannock Device ${ }^{\circledR}$ (Brannock Device Co, Inc., Liverpool, New York, USA) to ensure appropriate length and width, using the fitting protocol recommended by the footwear manufacturer [58]. Intervention group participants who wear flat insoles (or have been wearing contoured foot orthoses for more than three months) in their current footwear will be permitted to wear them in their study footwear, provided that the fit of the shoes is considered to be appropriate.

\section{Control group}

The control group will continue to receive regular podiatry treatment as clinically required. This will typically include toenail maintenance and scalpel debridement of hyperkeratotic lesions (corns and calluses). Upon completion of the trial, they will be provided with the same footwear used in the intervention group.

\section{Baseline assessments}

Participant characteristics will be collected by structured interview at the baseline assessment and will include age, sex, height, weight, waist circumference, hip circumference, country of birth, education, major medical

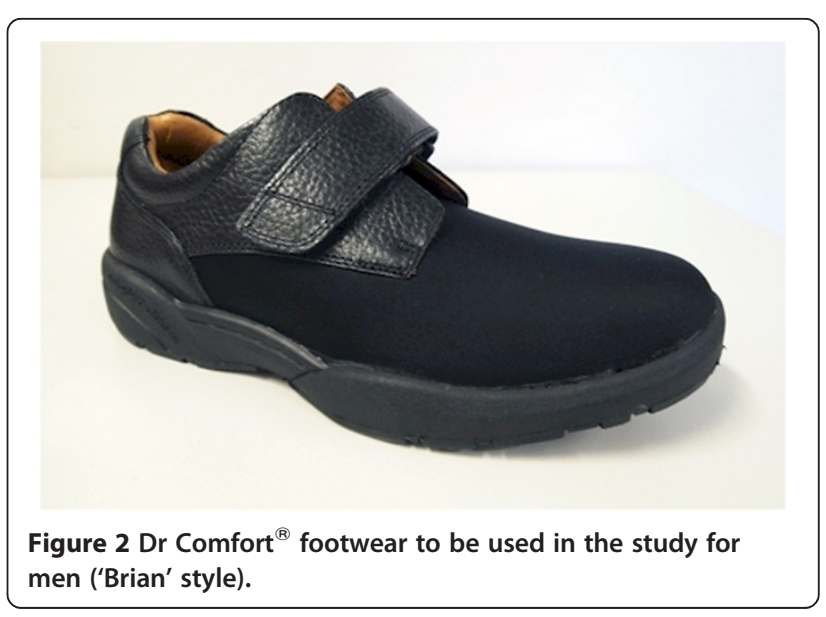




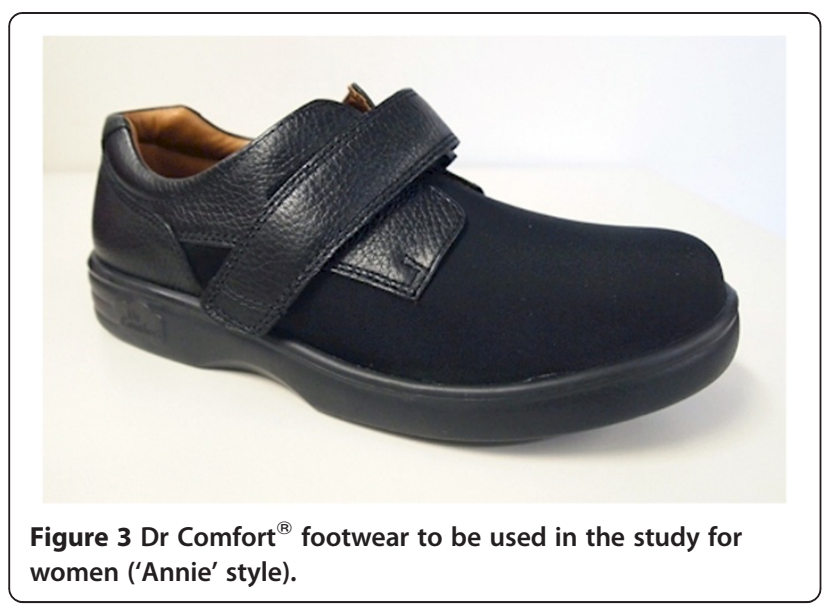

conditions, medications, use of walking aids and cigarette smoking history. The following questionnaires and clinical tests will also be administered:

(i) foot pain characteristics, including duration (in months) and location, using a standardized foot diagram;

(ii) presence and severity of hallux valgus, assessed using the Manchester Scale [59];

(iii) foot structure and presence of hyperkeratotic lesions (corns and calluses), documented with three-dimensional clinical photographs using the FotoScan $^{\text {TM }}$ 3D foot scanner (Precision 3D Limited, Weston-super-Mare, UK);

(iv) the Pain Catastrophizing Scale, a questionnaire containing 13 items reflecting elevated negative cognitive responses to pain [60];

(v) the short version of the Geriatric Depression Scale, a 15 -item depression screening tool that has been specifically validated in older people $[61,62]$;

(vi) footwear assessment, using selected components of the recently developed Footwear Assessment Tool [57], and;

(vii) physical activity levels, using the Incidental and Planned Activity Questionnaire [63].

In addition, participants allocated to the footwear intervention group will be asked to complete six $100 \mathrm{~mm}$ visual analogue scales to ascertain their perceptions of: (i) the immediate level of comfort of the footwear (using the anchors 'extremely uncomfortable' and 'extremely comfortable' [64]); (ii) the attractiveness of the footwear (using the anchors 'extremely unattractive' and 'extremely attractive'); (iii) how attractive they think other people would find the footwear (using the anchors 'extremely unattractive' and 'extremely attractive'); (iv) how well the shoes fit (using the anchors 'poorest fit possible' and 'best fit possible'); (v) how easy it is to put the shoes on and take them off (using the anchors 'most difficult as possible' and 'as easy as imaginable') and; (vi) how heavy the shoes are (using the anchors 'extremely light' and 'extremely heavy').

The perceived therapeutic value of the shoes will be assessed using responses to the statement 'I believe that the shoes provided to me can reduce the severity of my foot pain', with a five-point Likert scale ranging from 'strongly disagree' to 'strongly agree'. In addition, two $100 \mathrm{~mm}$ visual analogue scales will be used to ascertain expectations of pain reduction associated with the footwear, with the questions 'With your shoes, do you expect to have less or more pain in the skin of your feet and/or ankles, during activities like standing and/or walking?' and 'With your shoes, do you expect to have less or more pain in the muscles and joints of your feet and/or ankles, during activities like standing and/or walking?', both using the anchors 'much less' and 'much more'. These questions are derived from the Monitor Orthopedic Shoes questionnaire [65], and will be used to predict adherence to, and effectiveness of, the footwear intervention at the completion of the study.

\section{Primary outcome measure}

The primary outcome measure will be the pain domain of the Foot Health Status Questionnaire (FHSQ). The FHSQ consists of 13 questions reflecting four foot health-related domains: foot pain, foot function, footwear, and general foot health [66]. The FHSQ pain domain comprises four questions, with higher scores representing better foot health (that is, $100=$ best foot health and $0=$ worst foot health). The FHSQ pain domain demonstrates a high degree of content, criterion, and construct validity (Cronbach $\alpha=0.88$ ), high retest reliability (intraclass correlation coefficient $=0.86$ ) and has been used as an outcome measure in clinical trials for a range of foot disorders [67]. Previous research indicates that the minimal important difference for this measure is 12.5 points [68]. The FHSQ pain domain will be measured at baseline and at 4, 8, 12 and 16 weeks, with the primary endpoint being the 16 week score.

\section{Secondary outcome measures}

Secondary outcome measures will be documented at baseline and 16 weeks and will include:

(i) the function domain of the FHSQ [3];

(ii)the functional limitation, pain intensity and concern about appearance subscales of the MFPDI [49];

(iii) the number of DVA podiatry treatments required during the study period;

(iv) general health-related quality of life, assessed with the Short Form $12^{\circ}$ Version 2.0 [69];

(v) the number of falls experienced during the follow-up period; 
(vi) functional mobility, using the Timed Up and Go test [70];

(vii) presence of hyperkeratotic lesions (corns and calluses);

(viii) number of participants using co-interventions to relieve foot pain (such as oral non-steroidal antiinflammatory medications, topical medications and visits to other health-care practitioners), documented using a diary at 4, 8, 12 and 16 weeks, and;

(ix) participants' perception of overall treatment effect at week 16, assessed with the question 'Overall, how has your foot pain changed since the start of the study?' with a five-point Likert scale response ('marked worsening,' 'moderate worsening,' 'same', 'moderate improvement', or 'marked improvement'). For the purpose of analysis, this scale will then be dichotomized, where 'success' is defined as marked or moderate improvement on this scale.

\section{Sample size}

The sample size for the study has been calculated based on the pain domain of the FHSQ as the primary outcome measure [66]. Using a minimal important difference of 12.5 and a standard deviation of 23 obtained from a previous study [68], and assuming a $10 \%$ drop-out rate, the required sample size is 60 per group (power $=80 \%$ ). We believe that the $10 \%$ drop-out rate is reasonable, as our recent randomized controlled trial of a multifaceted podiatry intervention to prevent falls in older people had a drop-out rate of less than $5 \%$ over a much longer period of follow-up (12 months) [71]. The extra precision provided by covariate analysis was conservatively ignored when performing this calculation.

\section{Evaluation of adherence}

Adherence to the intervention will be documented at 4, 8, 12 and 16 weeks by asking participants on how many days (and for how many hours) they have worn their footwear, on average, in the past month.

\section{Complications and adverse events}

Complications and adverse events associated with the intervention are unlikely. However, the questionnaires at the 4, 8, 12 and 16 week follow-ups will provide participants with an opportunity to report any difficulties they have with the footwear, and all adverse events will be reported in the final manuscript.

\section{Statistical analysis}

Statistical analysis will be undertaken using SPSS version 20.0 (IBM Corp, NY, USA). All analyses will be conducted on an intention-to-treat principle using all randomized participants. Multiple imputation will be used to replace any missing data using five iterations, with age, baseline scores, and group allocation as predictors [72]. Demographic characteristics and baseline data will be summarized by descriptive statistics. Continuous data will be explored for normality using standard tests to satisfy the assumptions of parametric statistics. If data are found to be not normally distributed, transformation will be attempted using conventional techniques [73]. However, if data are still not normally distributed after transformation, non-parametric statistical tests will be used. Continuously-scored primary and secondary outcome measures with a normal distribution will be compared between groups using a linear regression technique with baseline scores, adjusted for by the analysis of covariance model $[74,75]$. Nominal and ordinal scaled data will be compared using chi-square analyses (or Fisher's exact test where appropriate) and Mann-Whitney $U$-tests, respectively. Effect sizes will be determined using Cohen's $d$ [76] for continuous data and relative risks for nominal data. Statistical significance for hypothesis tests will be set at the conventional level of $\alpha=0.05$.

\section{Discussion}

Footwear plays an important role in the maintenance of foot health and mobility in older people, and it has long been recognized that suboptimal footwear can be detrimental $[77,78]$. The provision of appropriate footwear, therefore, has considerable potential to reduce pain and improve health-related quality of life in this age-group. Although several studies have been undertaken to assess the effectiveness of footwear interventions in reducing foot pain in people with rheumatoid arthritis and other degenerative foot disorders [79-81], none have focused on the general veteran community. The study outlined in this protocol is, therefore, novel in that it targets a specific group of veterans (those ineligible for medical grade footwear) and uses generic, off-the-shelf footwear as the intervention.

Participants will be followed up at four-weekly intervals up to 16 weeks. This is longer than previous footwear intervention studies, which have employed 8 week [79] or 12 week $[80,81]$ follow-up periods. We are, therefore, confident that the duration of the study is sufficient to detect differences between the groups, if they exist. Furthermore, previous footwear studies have been limited to single baseline and follow-up assessments [79-81], whereas our study will incorporate five repeated measurements for the primary outcome measure (baseline, 4 weeks, 8 weeks, 12 weeks and 16 weeks). This will provide additional insight into the trajectory of any improvements in participants' foot pain. However, to address the issue of multiple testing of serial measurements [82,83], we have pre-specified 16 weeks as the primary end-point, and no 
statistical comparisons of the 4, 8 and 12 week scores will be undertaken.

Poor adherence is a well-recognized problem with footwear intervention studies [71,80,84] and has been attributed to the unique role of footwear as both an item of clothing and a health-related intervention [85]. We expect that the level of adherence to the off-the-shelf footwear in this study will be higher than that associated with medical grade footwear, due to better cosmesis. Furthermore, the initial postal invitation to take part in the study will specify the type of footwear that will be provided (accompanied by photographs), thereby providing veterans with an opportunity to decline participation on the basis of dissatisfaction with the appearance of the footwear. However, it is still likely that some participants will not be satisfied with the footwear either at the point of issue or during the follow-up period. The inclusion of baseline questionnaires addressing participants' immediate perceptions of the attractiveness, comfort and usability of the footwear may provide useful insights into their adherence during the trial.

There are three key limitations of the study design that warrant careful consideration. Firstly, due to the nature of the intervention, it will not be possible to blind participants to their group allocation, nor will it be possible to blind the investigators providing the intervention. Secondly, because the 'usual care' control group will receive no additional treatment until the completion of the study, there is a risk of what is often referred to as 'resentful demoralization'. That is, participants allocated to the control group may be resentful of not receiving the intervention, which may affect their adherence to the tasks required of them in the study and systematically influence their responses to the outcome measure questionnaires [86]. While this cannot be completely avoided, it may be offset to some extent by the fact that the control group participants will receive the intervention footwear at the completion of the trial, free of charge footwear that they would not otherwise be eligible for under the DVA program. One suggested approach to address this issue is to document participant group allocation preference as a prognostic variable and consider this in the statistical analysis [87]. However, this would not be appropriate in our study, as it is unlikely that any participants would prefer to wait 16 weeks for their footwear rather than receiving it at the commencement of the study. Finally, this trial has been pragmatically designed to ensure that the findings can be easily translated into 'real world' situations, where off-the-shelf footwear is most often purchased without clinical assessment or diagnosis from foot health professionals. As such, we have not restricted the inclusion criteria in relation to the underlying cause of foot pain, so it is possible that our sample will include some participants with conditions that may not be amenable to treatment with footwear.

\section{Trial status}

In summary, this study will be the first randomized controlled trial investigating the effectiveness of off-the-shelf footwear in reducing foot pain in Australian DVA clients ineligible for medical grade footwear. The study findings will be used to make evidence-based recommendations regarding the potential role of this intervention and will help inform the footwear provision component of the Rehabilitation Appliances Program of the DVA. Recruitment of participants commenced in October 2012 and final results are expected to be available in November 2013.

\section{Abbreviations \\ DVA: Department of Veterans' Affairs; FHSQ: Foot Health Status} Questionnaire; MFPDI: Manchester Foot Pain and Disability Index.

\section{Competing interests}

The authors declare that they have no competing interests.

\section{Authors' contributions}

HBM and NF conceived the idea and obtained funding for the study. HBM, NF and SEM designed the trial protocol. HBM drafted the manuscript. All authors have read and approved the final manuscript.

\section{Acknowledgements}

This study is funded by the Australian Department of Veterans' Affairs (Applied Research Program ID: ARP1031). HBM is currently a National Health and Medical Research Council Senior Research Fellow (ID: 1020925). We would like to thank Jane Fyfield (National Medical Advisor, Health Strategy and Gerontology, Australian Department of Veterans' Affairs) for her assistance, Matt Barkley and Craig Truscott (Vasyli Medical) for subsidizing the footwear used in the study and assisting with training of research assistants, and Maria Auhl and Sonja Ristevski (research assistants) for assisting with the final development of the protocol.

\section{Author details}

'Lower Extremity and Gait Studies Program, Faculty of Health Sciences, La Trobe University, Bundoora 3086, Victoria, Australia. ${ }^{2}$ Department of Podiatry, Faculty of Health Sciences, La Trobe University, Bundoora 3086, Victoria, Australia.

Received: 21 December 2012 Accepted: 4 April 2013

Published: 23 April 2013

\section{References}

1. Dunn JE, Link CL, Felson DT, Crincoli MG, Keysor JJ, McKinlay JB: Prevalence of foot and ankle conditions in a multiethnic community sample of older adults. Am J Epidemiol 2004, 159:491-498.

2. Menz HB, Tiedemann A, Kwan MMS, Plumb K, Lord SR: Foot pain in community-dwelling older people: an evaluation of the Manchester Foot Pain and Disability Index. Rheumatology 2006, 45:863-867.

3. Hill CL, Gill T, Menz HB, Taylor AW: Prevalence and correlates of foot pain in a population-based study: the North West Adelaide Health Study. J Foot Ankle Res 2008, 1:2.

4. White EG, Mulley GP: Footcare for very elderly people: a community survey. Age Ageing 1989, 18:275-278.

5. Black JR, Hale WE: Prevalence of foot complaints in the elderly. J Am Podiatr Med Assoc 1987, 77:308-311.

6. Cartwright A, Henderson G: More trouble with feet - a survey of the foot problems and chiropody needs of the elderly. London: Department of Health and Social Security, HMSO; 1986.

7. Benvenuti F, Ferrucci L, Guralnik JM, Gangemi S, Baroni A: Foot pain and disability in older persons: an epidemiologic survey. J Am Geriatr Soc 1995, 43:479-484. 
8. Gorter KJ, Kuyvenhoven MM, deMelker RA: Nontraumatic foot complaints in older people. A population-based survey of risk factors, mobility, and well-being. J Am Podiatr Med Assoc 2000, 90:397-402.

9. Bowling A, Grundy E: Activities of daily living: changes in functional ability in three samples of elderly and very elderly people. Age Ageing 1997, 26:107-114

10. Barr ELM, Browning C, Lord SR, Menz HB, Kendig H: Foot and leg problems are important determinants of functional status in community-dwelling older people. Disabil Rehabil 2005, 27:917-923.

11. Menz HB, Lord SR: The contribution of foot problems to mobility impairment and falls in older people. J Am Geriatr Soc 2001, 49:1651-1656.

12. Gabell A, Simons MA, Nayak USL: Falls in the healthy elderly: predisposing causes. Ergonomics 1985, 28:965-975.

13. Tinetti ME, Speechley M, Ginter SF: Risk factors for falls among elderly persons living in the community. New Eng J Med 1988, 319:1701-1707.

14. Koski K, Luukinen H, Laippala P, Kivela S-L: Physiological factors and medications as predictors of injurious falls by elderly people: a prospective population-based study. Age Ageing 1996, 25:29-38.

15. Leveille SG, Bean J, Bandeen-Roche K, Jones R, Hochberg M, Guralnik JM: Musculoskeletal pain and risk of falls in older disabled women living in the community. J Am Geriatr Soc 2002, 50:671-678.

16. Menz HB, Morris ME, Lord SR: Foot and ankle risk factors for falls in older people: a prospective study. J Gerontol A Biol Sci Med Sci 2006, 61A:M866-870.

17. Chen J, Devine A, Dick IM, Dhaliwal SS, Prince RL: Prevalence of lower extremity pain and its association with functionality and quality of life in elderly women in Australia. J Rheumatol 2003, 30:2689-2693.

18. Elewski BE: The effect of toenail onychomycosis on patient quality of life. Int J Dermatol 1997, 36:754-756.

19. Roseeuw D: Achilles foot screening project: preliminary results of patients screened by dermatologists. J Eur Acad Dermatol Venereol 1999, 12:56-9.

20. Schein JR, Gause D, Stier DM, Lubeck DP, Bates MM, Fisk R: Onychomycosis. Baseline results of an observational study. J Am Podiatr Med Assoc 1997, 87:512-519.

21. Thordarson DB, Ebramzadeh E, Rudicel SA, Baxter A: Age-adjusted baseline data for women with hallux valgus undergoing corrective surgery. J Bone Joint Surg Am 2005, 87A:66-75.

22. Lazarides SP, Hildreth A, Prassanna V, Talkhani I: Association amongst angular deformities in hallux valgus and impact of the deformity in health-related quality of life. Foot Ankle Surg 2005, 11:193-196.

23. Bergin SM, Munteanu SE, Zammit GV, Nikolopoulos N, Menz HB: Impact of first metatarsophalangeal joint osteoarthritis on health-related quality of life. Arthritis Care Res 2012, 64:1691-1698.

24. Irving DB, Cook JL, Young MA, Menz HB: Impact of chronic plantar heel pain on health-related quality of life. J Am Podiatr Med Assoc 2008, 98:283-289.

25. Brodie BS, Rees CL, Robins DJ, Wilson AFJ: Wessex Feet: a regional foot health survey, Volume I: The survey. Chiropodist 1988, 43:152-165.

26. Garrow AP, Silman AJ, Macfarlane GJ: The Cheshire Foot Pain and Disability Survey: a population survey assessing prevalence and associations. Pain 2004, 110:378-384.

27. Greenberg L, Davis H: Foot problems in the US. The National Health Interview Survey. J Am Podiatr Med Assoc 1990, 1993(83):475-483.

28. Menz HB, Morris ME: Determinants of disabling foot pain in retirement village residents. J Am Podiatr Med Assoc 2005, 95:573-579.

29. Leveille SG, Guralnik JM, Ferrucci L, Hirsch R, Simonsick E, Hochberg MC: Foot pain and disability in older women. Am J Epidemiol 1998, 148:657-665.

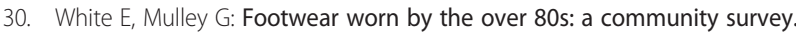
Clin Rehabil 1989, 3:23-25.

31. Munro BJ, Steele JR: Household-shoe wearing and purchasing habits - a survey of people aged 65 years and older. J Am Podiatr Med Assoc 1999, 89:506-514.

32. Jessup RL: Foot pathology and inappropriate footwear as risk factors for falls in a subacute aged-care hospital. J Am Podiatr Med Assoc 2007, 97:213-217.

33. Burns S, Leese G, McMurdo M: Older people and ill-fitting shoes. Postgrad Med J 2002, 78:344-346.
34. Nixon BP, Armstrong DG, Wendell C, Vazquez JR, Rabinovich Z: Do US veterans wear appropriately sized shoes? The Veterans Affairs shoe size selection study. J Am Podiatr Med Assoc 2006, 96:290-292.

35. Chaiwanichsiri D, Tantisiriwat N, Janchai S: Proper shoe sizes for Thai elderly. Foot 2008, 18:186-191.

36. Joyce P: Women and their shoes: attitudes, influences and behavior. $\mathrm{Br} J$ Podiat 2000, 3:111-115.

37. Seferin M, Van der Linden J: Protection or pleasure: female footwear. Work 2012, 41:290-294

38. Chantelau E, Gede A: Foot dimensions of elderly people with and without diabetes mellitus - a data basis for shoe design. Gerontology 2002, 48:241-244.

39. Mickle KJ, Munro BJ, Lord SR, Menz HB, Steele JR: Foot shape of older people: implications for shoe design. Footwear Sci 2010, 2:131-139.

40. Menz HB, Morris ME: Footwear characteristics and foot problems in older people. Gerontology 2005, 51:346-351.

41. Paiva de Castro A, Rebelatto JR, Aurichio TR: The relationship between foot pain, anthropometric variables and footwear among older people. Appl Ergon 2010, 41:93-97.

42. Frescos N, Menz HB: Foot health service provision by the Australian Department of Veterans' Affairs: do major podiatric medical interventions reduce the number of maintenance treatments? J Am Podiatr Med Assoc 2007, 97:469-474.

43. NHMRC: National statement on ethical conduct in human research. Canberra: Australian Government; 2007.

44. Ethical principles for medical research involving human subjects: http:// www.wma.net/en/30publications/10policies/b3/.

45. Schulz KF, Altman DG, Moher D: CONSORT 2010 statement: updated guidelines for reporting parallel group randomised trials. BMJ 2010, 340: c332.

46. Moher D, Hopewell S, Schulz KF, Montori V, Gøtzsche PC, Devereaux PJ, Elbourne D, Egger M, Altman DG: CONSORT 2010 explanation and elaboration: updated guidelines for reporting parallel group randomised trials. BMJ 2010, 340:c869.

47. Altman DG, Bland JM: Statistics notes. Treatment allocation in controlled trials: why randomise? BMJ 1999, 318:1209.

48. Altman DG, Bland JM: How to randomise. BMJ 1999, 319:703-704

49. Garrow AP, Papageorgiou AC, Silman AJ, Thomas E, Jayson MIV, Macfarlane $G J$ : Development and validation of a questionnaire to assess disabling foot pain. Pain 2000, 85:107-113.

50. Roddy E, Muller S, Thomas E: Defining disabling foot pain in older adults: further examination of the Manchester Foot Pain and Disability Index. Rheumatology 2009, 48:992-996.

51. Apelqvist JBK, van Houtum WH, Nabuurs-Franssen MH, Schaper $\mathrm{N}$ : International consensus and practical guidelines on the management and the prevention of the diabetic foot. Diabetes Metab Res Rev 2000 , 16(Suppl 1):S84-92.

52. Pfeiffer $\mathrm{E}$ : A short portable mental status questionnaire for the assessment of organic brain deficit in elderly patients. J Am Geriatr Soc 1975, 23:433-441.

53. Nancarrow S: Footwear suitability scale: a measure of shoe fit for people with diabetes. Australas J Podiatr Med 1999, 33:57-62.

54. Menz HB, Sherrington C: The Footwear Assessment Form: a reliable clinical tool for the evaluation of footwear characteristics of relevance to postural stability in older adults. Clin Rehabil 2000, 14:657-664.

55. Williams A: Footwear assessment and management. Podiatry Now 2006:S1-8

56. Healthy Footwear Guide: http://www.healthy-footwear-guide.com

57. Barton CJ, Bonanno D, Menz HB: Development and evaluation of a tool for the assessment of footwear characteristics. J Foot Ankle Res 2009, 2:10.

58. Dr Comfort Shoe Fitting Techniques: http://www.youtube.com/watch? $\mathrm{v}=$ XXkUZfZX7Ek

59. Garrow AP, Papageorgiou A, Silman AJ, Thomas E, Jayson Ml, Macfarlane GJ: The grading of hallux valgus. The Manchester Scale. J Am Podiatr Med Assoc 2001, 91:74-78.

60. Sullivan MJ, Bishop SR, Pivik J: The pain catastrophizing scale: development and validation. Psychol Assess 1995, 7:524-532.

61. Yesavage JA, Brink TL, Rose TL, Lum O, Huang V, Adey M, Leirer VO: Development and validation of a geriatric depression screening scale: a preliminary report. J Psychiatr Res 1983, 17:37-49. 
62. Sheikh Jl, Yesavage JA: Geriatric Depression Scale (GDS): recent evidence and development of a shorter version. In Clinical Gerontology: A Guide to Assessment and Intervention. Edited by Brink TL. New York: The Haworth Press; 1986:165-173.

63. Delbaere K, Hauer K, Lord SR: Evaluation of the incidental and planned activity questionnaire for older people. Br J Sports Med 2010 44:1029-1034

64. Mills K, Blanch P, Vicenzino B: Identifying clinically meaningful tools for measuring comfort perception of footwear. Med Sci Sports Exerc 2010, 42:1966-1971.

65. van Netten JJ, Hijmans JM, Jannink MJ, Geertzen JH, Postema K: Development and reproducibility of a short questionnaire to measure use and usability of custom-made orthopaedic shoes. J Rehabil Med 2009, 41:913-918.

66. Bennett $\mathrm{P}$, Patterson C, Wearing S, Baglioni T: Development and validation of a questionnaire designed to measure foot-health status. J Am Podiatr Med Assoc 1998, 88:419-428.

67. Riskowski JL, Hagedorn TJ, Hannan MT: Measures of foot function, foot health, and foot pain. Arthritis Care Res 2011, 63:S229-239.

68. Landorf KB, Radford JA, Hudson S: Minimal important difference (MID) of two commonly used outcome measures for foot problems. J Foot Ankle Res 2010, 3:7.

69. Ware J Jr, Kosinski M, Keller SD: A 12-item Short Form health survey: construction of scales and preliminary tests of reliability and validity. Med Care 1996, 34:220-233.

70. Podsialdo D, Richardson S: The timed 'Up and Go': a test of basic functional mobility for frail elderly persons. J Am Geriatr Soc 1991 39:142-148.

71. Spink MJ, Menz HB, Fotoohabadi MR, Wee E, Landorf KB, Hill KD, Lord SR: Effectiveness of a multifaceted podiatry intervention to prevent falls in community dwelling older people with disabling foot pain: randomized controlled trial. BMJ 2011, 342:d3411.

72. Sterne JA, White IR, Carlin JB, Spratt M, Royston P, Kenward MG, Wood AM Carpenter JR: Multiple imputation for missing data in epidemiological and clinical research: potential and pitfalls. BMJ 2009, 338:b2393.

73. Bland JM, Altman DG: Transforming data. BMJ 1996, 312:770.

74. Vickers AJ, Altman DG: Statistics notes: analysing controlled trials with baseline and follow-up measurements. BMJ 2001, 323:1123-1124.

75. Twisk J, Proper K: Evaluation of the results of a randomized controlled trial: how to define changes between baseline and follow-up. J Clin Epidemiol 2004, 57:223-228.

76. Cohen J: Statistical power analysis for the behavioral sciences. 2nd edition. Hillsdale, NJ: Erlbaum; 1988.

77. Finlay OE: Footwear management in the elderly care program. Physiotherapy 1986, 72:172-178.

78. Edelstein JE: If the shoe fits: footwear considerations for the elderly. Phys Occup Ther Geriatr 1987, 5:1-16.

79. Fransen $M$, Edmonds J: Off-the-shelf orthopedic footwear for people with rheumatoid arthritis. Arthritis Care Res 1997, 10:250-256.

80. Williams AE, Rome $K$, Nester CJ: A clinical trial of specialist footwear for patients with rheumatoid arthritis. Rheumatology 2007, 46:302-307.

81. Jannink MJ, Izerman MJ, Groothius-Oudshoorn K, Stewart RE, Groothoff JW, Lankhorst GJ: Use of orthopedic shoes in patients with degenerative disorders of the foot. Arch Phys Med Rehabil 2005, 86:687-692.

82. Matthews JN, Altman DG, Campbell MJ, Royston P: Analysis of serial measurements in medical research. BMJ 1990, 300:230-235.

83. Bender R, Lange S: Adjusting for multiple testing - when and how? J Clin Epidemiol 2001, 54:343-349.

84. Knowles EA, Boulton AJ: Do people with diabetes wear their prescribed footwear? Diabet Med 1996, 13:1064-1068.

85. Williams AE, Nester CJ, Ravey MI, Kottink A, Klapsing MG: Women's experiences of wearing therapeutic footwear in three European countries. J Foot Ankle Res 2010, 3:23.
86. Bradley C: Designing medical and educational intervention studies. A review of some alternatives to conventional randomized controlled trials. Diabetes Care 1993, 16:509-518.

87. Torgerson DJ, Klaber-Moffett J, Russell IT: Patient preferences in randomised trials: threat or opportunity? J Health Serv Res Policy 1996, 1:194-197.

doi:10.1186/1745-6215-14-106

Cite this article as: Menz et al:: Effectiveness of off-the-shelf footwear in reducing foot pain in Australian Department of Veterans' Affairs recipients not eligible for medical grade footwear: study protocol for a randomized controlled trial. Trials 2013 14:106.

\section{Submit your next manuscript to BioMed Central and take full advantage of:}

- Convenient online submission

- Thorough peer review

- No space constraints or color figure charges

- Immediate publication on acceptance

- Inclusion in PubMed, CAS, Scopus and Google Scholar

- Research which is freely available for redistribution
C Biomed Central 